\title{
Patients with an Inflamed Atherosclerotic Plaque have Increased Levels of Circulating Inflammatory Markers
}

\author{
Pavel Poredos ${ }^{1}$, Ana Spirkoska ${ }^{1}$, Luka Lezaic² ${ }^{2}$ Mojca Božič Mijovski ${ }^{1}$ and Mateja Kaja Jezovnik \\ ${ }^{1}$ Department of Vascular Disease, University Medical Centre Ljubljana, Ljubljana, Slovenia \\ ${ }^{2}$ Department of Nuclear Medicine, University Medical Centre Ljubljana, Ljubljana, Slovenia
}

Aim: Inflammation is highlighted in the pathogenesis and destabilization of atherosclerotic lesions. Noninvasive identification of inflammation of atherosclerotic lesions has been challenging. 18-Fluorodeoxyglucose (18F-FDG) positron emission tomography (PET) is a useful technique for detecting inflamed atherosclerotic plaques in vivo. However, it is time consuming, expensive, and accompanied by radiation. Therefore, we investigated the relationship between levels of circulating inflammatory markers and the degree of inflammation of atherosclerotic plaques shown by 18F-FDG uptake. We aimed to identify high-risk patients with inflamed, unstable atherosclerotic plaques on the basis of the determination of inflammatory markers.

Methods: The study included 37 patients, 21 with high-grade stenosis of internal carotid artery (ICA group) and 16 with occlusion of common femoral artery (CFA group), who underwent endarterectomy. Mean age of the study population was $69.43 \pm 6.2$ years. Eight out of 21 patients with ICA stenosis and all patients with CFA occlusion were symptomatic. In all patients before endarterectomy, 18F-FDG-PET imaging was performed and blood samples were obtained for determination of circulating inflammatory markers: high-sensitivity C-reactive protein (hsCRP), tumor necrosis factor alpha (TNF- $\alpha$ ), interleukins, and selectins. Both groups were compared with a sex- and age-matched control group composed of 27 healthy volunteers.

Results: 18 F-FDG uptake, calculated by target-to-background ratio (TBR) was not significantly different between the groups. Levels of inflammatory markers were elevated, and there were no significant differences between ICA and CFA groups, with an exception of interleukin 6 (IL-6) levels, which was higher in the ICA group $(3.2 \pm 2.5 \mathrm{ng} / \mathrm{L}$ vs. $1.8 \pm 1.3 \mathrm{ng} / \mathrm{L}, p<0.05)$. There was a positive interrelationship between 18F-FDG-PET and most of the systemic inflammatory markers: hsCRP $(r=0.417, p=0.010)$, IL-6 $(r=0.603, p<0.001)$, and TNF- $\alpha(r=0.374, p=0.023)$. However, correlation between 18F-FDG-PET and P-selectin, E-selectin, and t-PA was not found.

Conclusion: Our study showed that an interrelationship exists between the intensity of inflammatory process of atherosclerotic lesions shown by FDG uptake and circulating inflammatory markers. Therefore, the determination of circulating inflammatory markers can have a potential to identify individuals with unstable, inflamed atherosclerotic plaques.

Key words: Inflammation, Atherosclerotic plaques, Cardiovascular risk, Positron emission tomography

Copyright@2017 Japan Atherosclerosis Society

This article is distributed under the terms of the latest version of CC BY-NC-SA defined by the Creative Commons Attribution License.

\section{Introduction}

The detection of subclinical atherosclerotic lesions such as asymptomatic atherosclerotic plaques helps in

Address for correspondence: Mateja Kaja Jezovnik, Department of Vascular Disease, University Medical Centre Ljubljana, Ljubljana, Slovenia

E-mail: matejakaja@gmail.com

Received: January 19, 2016

Accepted for publication: April 5, 2016 identifying individuals in whom an atherosclerotic process is already present. These subjects have higher risk of cardiovascular events than those without atherosclerotic deterioration of the arterial wall ${ }^{1)}$. During the last decade, plaque structure composition rather than the degree of stenosis has proved to be a far more important trigger of thromboembolic events ${ }^{2,3)}$. Therefore, in addition to the detection of morphological characteristics of atherosclerotic plaques, recent research has focused on investigating the physiological 
processes, particularly inflammatory activity of atherosclerotic plaques, which help in identifying unstable atherosclerotic lesions and "vulnerable" patients. Evidence that inflammatory processes are pivotal in the development of unstable atherosclerotic lesions and related to complications is growing ${ }^{4)}$. Inflammation destabilizes atherosclerotic plaques and promotes their rupture by enzymatic degradation of their fibrous structure. Therefore, the inflammation of atherosclerotic plaque is associated with a significant increase in cardiovascular events ${ }^{5}$. Identification of unstable atherosclerotic plaques helps in identifying patients who are at a higher risk and need more intensive treatment of atherosclerosis risk factors.

There are different methods for detecting unstable atherosclerotic lesions. Invasive intravascular techniques are not acceptable for everyday clinical practice. Noninvasive identification of atherosclerotic processes has been a challenging task. High-resolution ultrasound is one of the noninvasive methods for identifying carotid atherosclerosis. However, it only provides evaluation and insight into the morphology of atherosclerotic lesions of the extracranial and some other peripheral arteries ${ }^{6}$. In addition, it gives no information regarding inflammation and metabolic status of the atherosclerotic lesions. Blockmans et al. were the first to report that 18-fluorodeoxyglucose (18F-FDG) positron emission tomography (PET) imaging is useful in detecting large vessel inflammation $^{7}$. Recent studies have demonstrated that PET imaging in combination with contrast-enhanced computed tomography $(\mathrm{CT})$ or magnetic resonance imaging enables visualization of $18 \mathrm{~F}-\mathrm{FDG}$ uptake in carotid plaques and that $18 \mathrm{~F}-\mathrm{FDG}$ accumulation is associated with the inflammation of atherosclerotic plaques related to macrophage infiltration ${ }^{8)}$. More recently, it was shown that 18 F-FDG-PET/CT enables very precise measurement of the inflammatory activity of atherosclerotic plaques in large- and medium-sized arteries ${ }^{9)}$.

In a previous study, we investigated the intensity of 18F-FDG uptake in the carotid and femoral arteries and compared it with the histological findings in the corresponding specimens obtained during carotid and femoral endarterectomy. We discovered a significant interrelationship between 18F-FDG uptake and the accumulation of inflammatory cells in carotid and femoral atherosclerotic lesions ${ }^{10)}$. 18F-FDG-PET imaging is relatively complicated, time consuming, and not completely noninvasive; therefore, investigators have been searching for other less complicated and noninvasive techniques for the identification of vessel wall inflammation. As inflammation of an individual organ system usually triggers a systemic inflammatory response, we expected that in patients with atherosclerotic inflammatory lesions of vessel wall, inflammatory markers in systemic blood, particularly those which indicate inflammation of the arterial wall (interleukins), would be increased. Therefore, the aim of the study was to investigate the relationship between the inflammation of carotid and femoral atherosclerotic plaques determined by PET/CT and levels of systemic inflammatory markers and to identify patients at high risk for cardiovascular events on the basis of the investigation of blood constituents.

\section{Materials and Methods}

\section{Study Design and Participants}

The study included 37 patients, 21 with highgrade stenosis of the internal carotid artery (ICA) ( $\geq 70 \%$ luminal narrowing) and 16 with occlusion of the common femoral artery (CFA), who underwent endarterectomy of ICA or CFA, respectively. There were $24(65 \%)$ males and $13(35 \%)$ females. The mean age of the study population was $69.43 \pm 6.2$ years, and there were no statistically significant differences in the age between males and females (68.9 vs. 70.7 years, $p=0.470$ ). Eight out of the 21 patients with stenosis of the ICA were symptomatic: three had unilateral amaurosis fugax, four suffered a transitory ischemic attack, and one suffered a hemispheric stroke in the carotid territory of interest. All patients with occlusion of CFA were symptomatic; four of them fulfilled the criteria for critical limb ischemia (rest pain, ankle brachial pressure $<50 \mathrm{mmHg}$ ), and the others had limiting intermittent claudication. The diagnosis was first confirmed by Duplex ultrasonography and then by CT angiography. Both groups were compared with a sex- and age-matched control group composed of 27 healthy volunteers.

The study was conducted according to the ethical principles stated in the Declaration of Helsinki. The study protocol was approved by the National Ethical Committee, and all patients provided written consent.

\section{F-FDG-PET Imaging}

Before the operative procedure (endarterectomy), 18F-FDG-PET imaging was performed in all patients. After at least $12 \mathrm{~h}$ of fasting, the serum glucose level was checked using a finger stick, and a 20 -gauge intravenous (iv) line was inserted to administer the radiotracer. The study patients received $370 \mathrm{MBq}$ of $18 \mathrm{~F}$ FDG intravenously. One hour after iv 18F-FDG administration, low-dose CT imaging of the region of interest was performed $(120 \mathrm{keV}, 50 \mathrm{~mA}$, and slice thickness $3 \mathrm{~mm}$ ) from the external auditory meatus to 
Table 1. The clinical characteristics and medications of patients with ICA and CFA atherosclerotic lesions who underwent endarterectomy (values are given as means \pm SD or absolute numbers and percentages).

\begin{tabular}{|c|c|c|c|c|}
\hline & $\begin{array}{c}\text { CFA } \\
(n=16)\end{array}$ & $\begin{array}{c}\text { ICA } \\
(n=21)\end{array}$ & $\begin{array}{l}\text { Whole group } \\
\qquad(n=37)\end{array}$ & $p$ \\
\hline Age (years) & $69.6 \pm 6.9$ & $70.4 \pm 5.9$ & $69.5 \pm 6.8$ & NS \\
\hline Sex $(M)$ & $12(75 \%)$ & $12(57 \%)$ & $24(65 \%)$ & NS \\
\hline Arterial hypertension & $11(68 \%)$ & $20(95 \%)$ & $31(84 \%)$ & NS \\
\hline Diabetes mellitus & $6(37 \%)$ & $8(38 \%)$ & $14(38 \%)$ & NS \\
\hline Hyperlipidemia & $10(63 \%)$ & $19(90 \%)$ & $29(78 \%)$ & NS \\
\hline Smoking & $4(25 \%)$ & $4(19 \%)$ & $8(22 \%)$ & $<0.01$ \\
\hline Former smoker & $3(19 \%)$ & $1(5 \%)$ & $4(11 \%)$ & $<0.01$ \\
\hline Statins & $10(63 \%)$ & $18(86 \%)$ & $28(76 \%)$ & NS \\
\hline Antidiabetics & $8(50 \%)$ & $8(38 \%)$ & $16(43 \%)$ & NS \\
\hline - Insulin & $4(25 \%)$ & $2(10 \%)$ & $6(16 \%)$ & NS \\
\hline - Peroral drugs & $3(19 \%)$ & $4(19 \%)$ & $7(19 \%)$ & NS \\
\hline - Diet & $3(19 \%)$ & $2(10 \%)$ & $5(14 \%)$ & NS \\
\hline Antihypertensive drugs & $12(75 \%)$ & $16(76 \%)$ & $28(76 \%)$ & NS \\
\hline
\end{tabular}

Abbreviations: ICA - internal carotid artery, CFA - common femoral artery, NS - nonsignificant

the aortic arch level for the carotid arteries and from the aortic bifurcation to the popliteal fossa for the iliofemoral arteries for localization and attenuation correction. CT was immediately followed by 3D PET imaging (Biograph mCT PET/CT scanner, Siemens Healthcare, Erlangen, Germany) of the same region ${ }^{11)}$. Combination of PET and CT imaging (PET/CT) was performed by reviewing on a workstation (Segami Oasis, Segami Corporation, Columbia, MD, USA) as previously described ${ }^{12)}$. PET images were reconstructed using an ordered-subset expectation maximization algorithm (two iterations, 21 subsets) with matching final slice thickness (voxel size $4.07 \mathrm{~mm}$ ). The uptake value of PET imaging with $18 \mathrm{~F}-\mathrm{FDG}$ was estimated by the standardized uptake value (SUV) of the artery of interest and venous blood to calculate the target-tobackground ratio (TBR), which was calculated by dividing the arterial plaque maximal SUV by the venous blood average SUV.

\section{Laboratory Methods}

Laboratory studies in all patients included markers of inflammation [high-sensitivity C-reactive protein (hsCRP), interleukin 6 (IL-6), and tumor necrosis factor alpha (TNF- $\alpha$ )], markers of endothelial damage (P-selectin and E-selectin), and hemostatic factors [(tissue plasminogen activator antigen ( $t-P A$ $\mathrm{Ag})$ ]. In all patients, blood samples were obtained at the time of endarterectomy and repeated $1-2$ years after the procedure. The concentrations of laboratory parameters were measured by enzyme-linked immunosorbent assay (ELISA) using commercially available kits according to manufacturer instructions.

\section{Statistical Analysis}

Continuous variables were expressed as means \pm standard deviation (SD). For comparison of the continuous variables, the Mann-Whitney $U$ test was used, and the chi-square or Fisher's exact test was used for comparison of categorical data. Pearson's correlation coefficient method was used to assess the correlation between PET maximal TBR and levels of circulating markers. Statistical analyses were performed using IBM SPSS Statistics for Windows, Version 23.0. Armonk, NY: IBM Corp., USA.

\section{Results}

Thirty-seven patients and 27 healthy controls were enrolled in the study: 21 with ICA stenosis (8 symptomatic and 13 asymptomatic) and 16 with occlusion of CFA. The study included patients who successfully underwent endarterectomy; their characteristics are shown in Table $\mathbf{1}$. There were no significant differences in age between patients with carotid and femoral atherosclerosis $(p=0.47)$ or between males and females $(p=0.43)$.

All patients were at high risk of cardiovascular events; three of them had only one risk factor of atherosclerosis, and in the others, two or more classical risk factors were present. Arterial hypertension was most frequently present, followed by hypolipoproteinemia, diabetes mellitus, and smoking. There were no significant differences in the presence of risk factors 
Table 2. Values of systemic inflammatory markers and t-PA Ag in patients who underwent endarterectomy of ICA or CFA in comparison to healthy controls (values are given as mean $\pm \mathrm{SD}$ )

\begin{tabular}{lccccc}
\hline \multicolumn{1}{c}{ Parameter } & $\begin{array}{c}\text { Controls } \\
(n=27)\end{array}$ & $\begin{array}{c}\text { ICA } \\
(n=21)\end{array}$ & $\begin{array}{c}\text { CFA } \\
(n=16)\end{array}$ & $\begin{array}{c}p \\
\text { (ICA vs CFA) }\end{array}$ & $\begin{array}{c}p \\
\text { (controls vs. ICA+CFA) }\end{array}$ \\
\hline hsCRP $(\mathrm{mg} / \mathrm{L})$ & $2.48 \pm 1.1$ & $2.5 \pm 1.5$ & $2.7 \pm 1.7$ & 0.23 & 0.43 \\
IL-6 $(\mathrm{ng} / \mathrm{L})$ & $1.93 \pm 1.4$ & $3.2 \pm 2.5$ & $1.8 \pm 1.3$ & $<0.05$ & $<0.05$ \\
TNF- $\alpha(\mathrm{ng} / \mathrm{L})$ & $1.65 \pm 0,5$ & $2.1 \pm 0.6$ & $2.3 \pm 0.7$ & 0.43 & 0.04 \\
P-selectin $(\mu \mathrm{g} / \mathrm{L})$ & $39.10 \pm 16,2$ & $48.5 \pm 20.3$ & $37.6 \pm 17.4$ & 0.08 & 0.38 \\
E-selectin $(\mu \mathrm{g} / \mathrm{L})$ & $10.20 \pm 4,1$ & $12.8 \pm 2.4$ & $10.6 \pm 2.8$ & 0.15 & 0.67 \\
t-PA Ag $(\mu \mathrm{g} / \mathrm{L})$ & $12.6 \pm 3,7$ & $12.6 \pm 5.4$ & $14.2 \pm 4.4$ & 0.23 & 0.43 \\
\hline
\end{tabular}

Abbreviations: ICA - internal carotid artery, CFA - common femoral artery, hsCRP - high sensitivity C-reactive protein, IL-6 - interleukin 6, TNF- $\alpha$ - tumour necrosis factor alpha, t-PA Ag - tissue plasminogen activator antigen.

Table 3. Values of 18F-FDG-PET evaluation in patients with ICA and CFA atherosclerotic lesions (values are given as means $\pm \mathrm{SD}$ )

\begin{tabular}{lcccc}
\hline $\begin{array}{c}\text { ICA stenosis } \\
(n=21)\end{array}$ & $\begin{array}{c}\text { CFA occlusion } \\
(n=16)\end{array}$ & $\begin{array}{c}\text { Whole group } \\
(n=37)\end{array}$ & $p$ \\
\hline Maximal TBR & $1.9 \pm 0.4$ & $1.8 \pm 0.3$ & $1.8 \pm 0.4$ & 0.177 \\
\hline
\end{tabular}

between patients with carotid and femoral atherosclerosis, with the exception of smokers or former smokers who represented $50 \%$ of patients in the CFA group and $16 \%$ of the subjects with carotid stenosis (Table 1). In the control group, five subjects had increased blood pressure and four had dyslipidemia.

Table 2 shows the levels of systemic inflammatory markers and t-PA Ag values of the investigated patient groups and controls. With an exception of IL-6, which was higher in patients with carotid stenosis, there were no significant differences in the levels of inflammatory markers between patients with ICA stenosis and patients with occluded CFA. Between controls and both patient groups, significant differences were detected in IL- 6 and TNF- $\alpha$ levels. Both markers were significantly lower in healthy controls compared with the patients. The values of 18F-FDG-PET were also comparable between the investigated patient groups (Table 3). In subjects with ICA stenosis, maximal TBR was not significantly different compared with patients with CFA occlusion.

Table 4 presents the interrelationship between 18F-FDG-PET and levels of systemic inflammatory markers. A close relationship between the 18F-FDG uptake presented as maximal TBR and circulating inflammatory markers, such as IL6, hsCRP, and TNF- $\alpha$, was proven and a borderline significant interrelationship was indicated between P-selectin levels and 18F-FDG-PET.

There were no statistically significant differences
Table 4. Interrelationship between 18F-FDG-PET and systemic inflammatory markers ( $r$ : Pearson coefficient of correlation, $p$-value).

\begin{tabular}{llrr}
\hline & & $r$ & \multicolumn{1}{c}{$p$} \\
\hline Maximal TBR: & hsCRP & 0.417 & 0.010 \\
& IL-6 & 0.603 & $<0.001$ \\
& TNF- $\alpha$ & 0.374 & 0.023 \\
& P-selectin & 0.302 & 0.069 \\
& E-selectin & 0.157 & 0.354 \\
& t-PA Ag & 0.110 & 0.518 \\
\hline
\end{tabular}

in the levels of systemic inflammatory markers determined before endarterectomy compared with the levels after endarterectomy in the ICA and CFA groups, respectively (Table 5).

\section{Discussion}

The structure of atherosclerotic plaques is most probably an essential element in thromboembolic complications of atherosclerosis, and inflammation may play an important role in plaque vulnerability or stability. Inflammatory processes within the arterial wall were initiated mainly in response to endogenously modified structures, particularly oxidized lipoproteins, which lead to further alteration of the vascular wall and promote disease progression ${ }^{13)}$. The associations between inflammation of atherosclerotic lesion and 
Table 5. Systemic inflammatory markers in ICA and CFA groups, respectively before (B) and after (A) endartectomy.

\begin{tabular}{lccccc}
\hline \multicolumn{1}{c}{ Parameter } & $\begin{array}{c}\text { ICA-B } \\
(n=21)\end{array}$ & $\begin{array}{c}\text { ICA-A } \\
(n=15)\end{array}$ & $\begin{array}{c}\text { CFA-B } \\
(n=16)\end{array}$ & $\begin{array}{c}\text { CFA-A } \\
(n=12)\end{array}$ & $\begin{array}{c}p \\
(\text { ICA + CFA) B vs (ICA+CFA) A }\end{array}$ \\
\hline hsCRP (mg/L) & $2.5 \pm 1.5$ & $2.4 \pm 1.1$ & $2.7 \pm 1.7$ & $2.4 \pm 1.3$ & 0.43 \\
IL-6 (ng/L) & $3.2 \pm 2.5$ & $1.8 \pm 1.0$ & $1.8 \pm 1.3$ & $1.7 \pm 1.4$ & 0.06 \\
TNF- $\alpha(\mathrm{ng} / \mathrm{L})$ & $2.1 \pm 0.6$ & $1.9 \pm 0.8$ & $2.3 \pm 0.7$ & $2.2 \pm 0.9$ & 0.27 \\
P-selectin $(\mu \mathrm{g} / \mathrm{L})$ & $48.5 \pm 20.3$ & $42.7 \pm 19.4$ & $36.7 \pm 17.4$ & $38.1 \pm 16.5$ & 0.41 \\
\hline
\end{tabular}

Abbreviations: ICA - internal carotid artery stenosis, CFA - common femoral artery occlusion, hsCRP - high sensitivity C-reactive protein, IL-6 interleukin 6, TNF- $\alpha$ - tumour necrosis factor alpha

cardiovascular events ${ }^{14)}$ have led to the development of techniques that can measure the intensity of inflammation of the vessel wall. As shown in our previous study and in other studies, 18F-FDG-PET/CT is able to detect and quantify inflammation within carotid and femoral atherosclerotic lesions ${ }^{10)}$. 18F-FDG uptake in the arteries shows a good correlation with different risk factors of atherosclerosis and correlates with macrophage quantities within the atherosclerotic plaques ${ }^{12)}$. However, its specificity is relatively low because an increased 18F-FDG glucose uptake is also present in other diseases with high metabolic processes as well as in malignant diseases.

Recently, various circulating markers have been evaluated as indicators of plaque inflammation. In addition, their sensitivity to reveal which patient may benefit from intensified medical treatment that stabilizes atherosclerotic plaques has been evaluated ${ }^{15)}$.

Circulating markers, which are indicators of vessel wall inflammation, are of particular interest. Some cytokines such as IL-6 and IL-18 have been identified to play a role in the late stages of atherosclerotic lesion development, causing thinning of the extracellular matrix cap, which makes plaques prone to rupture ${ }^{16)}$. Levels of IL- 6 were also shown to be associated with the angiotensin II expression and may contribute to the inflammatory processes within the vascular wall and development of cardiovascular complications ${ }^{17)}$.

In our study, the level of IL-6 was significantly correlated to $18 \mathrm{~F}-\mathrm{FDG}$-PET uptake in carotid and femoral atherosclerotic lesions, which indicates that increased circulating levels of IL- 6 are most probably the consequence of inflamed atherosclerotic plaques in the investigated arterial segments. Particularly, carotid stenotic atherosclerotic lesions usually express a more intensive inflammatory process than occlusive lesions of femoral arteries ${ }^{10)}$. Atherosclerotic plaques, particularly, unstable ones, are metabolically more active than thrombotic material, which is a cause of occlusion of femoral artery. Therefore, the finding that patients with ICA stenosis have higher levels of IL-6 (indicator of vessel wall inflammation) than patients with thrombotic occlusion of CFA supports the hypothesis that the inflammation is probably more intensive in nonocclusive carotid lesions.

The involvement of IL- 6 in atherogenesis is also supported by findings that IL- 6 modulates lipid homeostasis, vascular remodeling, and plaque inflammation in atherogenesis ${ }^{18)}$. Further, high levels of IL-6 have been shown to be associated with lower echogenicity of carotid plaques, suggesting a link between inflammation and the potential risk of plaque ruptures ${ }^{19)}$. However, IL-6 level elevation is nonspecific and is also secreted by $\mathrm{T}$ cells during infection ${ }^{20)}$.

In our study, the level of hsCRP was also significantly correlated with 18F-FDG-PET values, which again indicates that inflamed atherosclerotic plaques may cause a systemic inflammatory response. hsCRP is a well-known circulating marker of atherosclerosis, and its levels correlate with the presence of cardiovascular disease. hsCRP is most probably directly involved in atherogenesis; it binds to a modified form of low-density lipoprotein (LDL), and when aggregated, hsCRP can also bind to native $\mathrm{LDL}^{21)}$.

Therefore, different mechanisms have been identified by which hsCRP modulates and evolves the process of atherosclerosis. However, hsCRP is a much less specific indicator of vessel wall inflammation than ILs. In our study, circulating levels of IL-6 and hsCRP were both increased and correlated with the intensity of inflammation of atherosclerotic plaques shown by 18F-FDG-PET uptake. Similar findings were reported by the Yamagami group, who discovered that increased levels of IL-6 and hsCRP appear to be associated with lower echogenicity of carotid plaques, which indicates inflammation of atherosclerotic lesions ${ }^{22)}$.

TNF- $\alpha$, a pleotropic cytokine exerting both inflammatory and cell death modulatory activity, is also one of the inflammatory markers. It is thought to play a role in the pathogenesis of atherosclerosis, particularly myocardial ischemia, reperfusion injury, and heart failure ${ }^{23)}$. Plasma concentration of TNF- $\alpha$ is also 
persistently elevated among postmyocardial infarction patients and is related to increased risk of cardiovascular events. It again indicates that a persistent inflammatory process is associated with increased vascular risk $^{24)}$.

TNF- $\alpha$ alters endothelial and vascular smooth muscle cell function as well as endothelial and blood cell interaction. It also promotes the expression of adhesion molecules on endothelial cells and initiation of an inflammatory cascade inside the arterial wall ${ }^{25}$. TNF- $\alpha$ may also interfere with triglycerides and cholesterol metabolic pathways. It promotes hepatic cholesterol synthesis and decreases high-density lipoprotein cholesterol ${ }^{26)}$.

In our study, TNF- $\alpha$ was significantly related to the intensity of inflammation of the atherosclerotic plaques, indicated by increased uptake of 18F-FDGPET. This finding confirms that patients with inflamed atherosclerotic plaques have increased levels of inflammatory markers, which are most probably a consequence of increased synthesis and release of these markers by the inflamed atherosclerotic lesions. Nevertheless, most of these patients were treated with statins, which inhibit inflammatory response and stabilize atherosclerotic plaques. In our study, inflammation was most probably not completely inhibited and consequently circulating levels of biomarkers of inflammation remained elevated.

Measurement of circulating markers after 1-2 years after the endartectomy showed that the levels of most of the inflammatory markers were not significantly different compared with the preoperative period. It indicates that atherosclerosis is a systemic disease where patients with atherosclerotic plaques or occlusions in one region most probably also have atherosclerotic lesions in other parts of the arterial system. Elimination of a single atherosclerotic lesion with endartectomy does not cure the disease; therefore, patients are at a high risk of cardiovascular events in other parts of the circulatory system. Borderline decrease of IL- 6 levels after operative procedure indicates that this inflammatory marker is an indicator of vessel wall alteration and inflammation. Therefore, in addition to local surgical intervention, these patients need intensive antiatherosclerotic treatment and prevention including antiplatelet and lipid-lowering drugs, which stabilize atherosclerotic lesions and decrease inflammation in atherosclerotic plaques. Tahara et al. showed that 18F-FDG-PET signals in atherosclerotic lesions were suppressed after a 3-months treatment with a statin ${ }^{27)}$. In our study, most of the patients were treated with statins; however, less than half of them had reached target levels of cholesterol. Therefore, in our study, it was not possible to exactly estimate the influence of the statins on the 18F-FDG uptake and levels of circulating inflammatory markers.

\section{Methodological Consideration}

Circulating inflammatory markers are nonspecific, and an increase in their levels can be caused by different diseases, particularly, systemic inflammatory diseases and traumatic tissue damage. Therefore, the results should be interpreted with caution as there is still no definitive answer to whether increased levels of these markers are the cause or the consequence of arterial wall inflammation in atherosclerosis. After the exclusion of other causes, a moderate or mild increase of some markers, particularly ILs, may indicate vessel wall inflammation.

In our study, inflammation of atherosclerotic plaques was investigated through 18F-FDG uptake combined with contrast-enhanced CT. For the quantification of 18F-FDG uptake in an atherosclerotic plaque, measurement of the standardized uptake value of the artery of interest was used, and background 18F-FDG uptake of the blood was measured to calculate maximal $\mathrm{TBR}^{9)}$. There are several methodological considerations regarding the methodology of arterial imaging using 18F-FDG-PET. Recent evidence indicates that maximal TBR is probably the most reproducible value for the measurement of $18 \mathrm{~F}-\mathrm{FDG}$ uptake in the carotid arteries ${ }^{11}$. However, because FDG is taken up by any metabolically active tissue, concerns have been raised about the specificity of this tracer for imaging inflammatory cells. Further, blood glucose level can influence FDG uptake, particularly in diabetic patients ${ }^{28)}$. On the other hand, studies have shown that FDG uptake is significantly correlated with the components of metabolic syndrome ${ }^{29)}$. Various drugs can also influence FDG uptake, particularly anti-inflammatory drugs and statins. In our study, all these factors that can potentially influence FDG uptake with the exception of statins were excluded.

\section{Conclusion}

The evidence supports the central role of inflammation in atherogenesis. Consequently, it is expected that patients with inflamed atherosclerotic plaques have increased levels of circulating markers, particularly hsCRP and ILs. Our study confirmed that the levels of some circulating markers (IL-6, TNF- $\alpha$, and hsCRP) that indicate inflammation of the vessel wall were correlated with the intensity of the inflammatory processes in atherosclerotic plaques, as shown by 18F-FDG-PET. In spite of the fact that these markers are nonspecific indicators of vessel wall inflammation, 
after exclusion of other inflammatory processes, determination of circulating markers has the potential to identify individuals with unstable, inflamed atherosclerotic plaques who are at the highest risk for future cardiovascular events and may benefit from more aggressive preventive interventions.

\section{Funding}

This study was realized with the support of the research program P3-0308 financed by the Slovenian Research Agency.

\section{Conflict of Interest}

None.

\section{References}

1) Poredoš P. Peripheral arterial occlusive disease increases the risk of perioperative complications. Vasa. 2014; 43: 401-403

2) Naghavi M, Libby P, Falk E, Casscells SW, Litovsky S, Rumberger J. From vulnerable plaque to vulnerable patient: a call for new definitions and risk assessment strategies: Part I. Circulation. 2003; 108: 1664-1672

3) Schwartz SM, Galis ZS, Rosenfeld ME, Falk E. Plaque rupture in humans and mice. Arterioscler Thromb Vasc Biol. 2007; 27: 705-713

4) Rudd JH, Myers KS, Bansilal S, Machac J, Rafique A, Farkouh M. (18)Fluorodeoxyglucose positron emission tomography imaging of atherosclerotic plaque inflammation is highly reproducible: implications for atherosclerosis therapy trials. J Am Coll Cardiol. 2007; 50: 892-896

5) Hubalewska-Dydejczyk A, Stompór T, Kalembkiewicz M, Krzanowski M, Mikolajczak R, Sowa-Staszczak A. Identification of inflamed atherosclerotic plaque using 123 I-labeled interleukin-2 scintigraphy in high-risk peritoneal dialysis patients: a pilot study. Perit Dial Int. 2009; 29: 568-574

6) Nighoghossian N, Derex L, Douek P. The vulnerable carotid artery plaque: current imaging methods and new perspectives. Stroke. 2005; 36: 2764-2772

7) Blockmans D, Maes A, Stroobants S, Nuyts J, Bormans $G$, Knockaert D. New arguments for a vasculitic nature of polymyalgia rheumatica using positron emission tomography. Rheumatology (Oxford). 1999; 38: 444-447

8) Tawakol A, Migrino RQ, Bashian GG, Bedri S, Vermylen $\mathrm{D}$, Cury RC. In vivo $18 \mathrm{~F}$-fluorodeoxyglucose positron emission tomography imaging provides a noninvasive measure of carotid plaque inflammation in patients. $\mathrm{J}$ Am Coll Cardiol. 2006; 48: 1818-1824

9) Mehta NN, Torigian DA, Gelfand JM, Saboury B, Alavi A. Quantification of atherosclerotic plaque activity and vascular inflammation using [18-F] fluorodeoxyglucose positron emission tomography/computed tomography (FDGPET/CT). J Vis Exp. 2012(63): e3777

10) Jezovnik MK, Zidar N, Lezaic L, Gersak B, Poredos P.
Identification of inflamed atherosclerotic lesions in vivo using PET-CT. Inflammation. 2014; 37: 426-434

11) Rudd JH, Myers KS, Bansilal S, Machac J, Pinto CA, Tong C. Atherosclerosis inflammation imaging with $18 \mathrm{~F}$ FDG PET: carotid, iliac, and femoral uptake reproducibility, quantification methods, and recommendations. J Nucl Med. 2008; 49: 871-878

12) Rudd JH, Warburton EA, Fryer TD, Jones HA, Clark JC, Antoun N. Imaging atherosclerotic plaque inflammation with [18F]-fluorodeoxyglucose positron emission tomography. Circulation. 2002; 105: 2708-2711

13) Mallat Z, Taleb S, Ait-Oufella H, Tedgui A. The role of adaptive $\mathrm{T}$ cell immunity in atherosclerosis. J Lipid Res. 2009; 50: S364-369

14) Libby P. Inflammation in atherosclerosis. Nature. 2002; 420: 868-874

15) Sillesen H. What does 'best medical therapy' really mean? Eur J Vasc Endovasc Surg. 2008; 35: 139-144

16) Mallat Z, Corbaz A, Scoazec A, Besnard S, Lesèche G, Chvatchko Y. Expression of interleukin-18 in human atherosclerotic plaques and relation to plaque instability. Circulation. 2001; 104: 1598-1603

17) Schieffer B, Schieffer E, Hilfiker-Kleiner D, Hilfiker A, Kovanen PT, Kaartinen M. Expression of angiotensin II and interleukin 6 in human coronary atherosclerotic plaques: potential implications for inflammation and plaque instability. Circulation. 2000; 101: 1372-1378

18) Schieffer B, Selle T, Hilfiker A, Hilfiker-Kleiner D, Grote $\mathrm{K}$, Tietge UJ. Impact of interleukin- 6 on plaque development and morphology in experimental atherosclerosis. Circulation. 2004; 110: 3493-3500

19) Grønholdt ML, Nordestgaard BG, Bentzon J, Wiebe BM, Zhou J, Falk E. Macrophages are associated with lipidrich carotid artery plaques, echolucency on Bmode imaging, and elevated plasma lipid levels. J Vasc Surg. 2002; 35: $137-145$

20) Reikerås O, Borgen P. Activation of markers of inflammation, coagulation and fibrinolysis in musculoskeletal trauma. PLoS One. 2014; 9: e107881

21) Singh SK, Suresh MV, Voleti B, Agrawal A. The connection between Creactive protein and atherosclerosis. Ann Med. 2008; 40: 110-120

22) Yamagami H, Kitagawa K, Nagai Y, Hougaku H, Sakaguchi M, Kuwabara K, et al. Higher levels of interleukin-6 are associated with lower echogenicity of carotid artery plaques. Stroke. 2004; 35: 677-681

23) Kleinbongard P, Heusch G, Schulz R. TNFalpha in atherosclerosis, myocardial ischemia/reperfusion and heart failure. Pharmacol Ther. 2010; 127: 295-314

24) Ridker PM, Rifai N, Pfeffer M, Sacks F, Lepage S, Braunwald E. Elevation of tumor necrosis factor-alpha and increased risk of recurrent coronary events after myocardial infarction. Circulation. 2000; 101: 2149-2153

25) Ross R. Atherosclerosis--an inflammatory disease. N Engl J Med. 1999; 340: 115-126

26) Popa C, Netea MG, van Riel PL, van der Meer JW, Stalenhoef AF. The role of TNF-alpha in chronic inflammatory conditions, intermediary metabolism, and cardiovascular risk. J Lipid Res. 2007; 48: 751-762

27) Tahara N, Kai H, Ishibashi M, Nakaura H, Kaida H, Baba K, Hayabuchi N, Imaizumi T. Simvastatin attenu- 
ates plaque inflammation: evaluation by fluorodeoxyglucose positron emission tomography. J Am Coll Cardiol 2006; 48: 1825-1831

28) Silvola JM, Saraste A, Laitinen I, Savisto N, Laine VJ, Heinonen SE. Effects of age, diet, and type 2 diabetes on the development and FDG uptake of atherosclerotic plaques. JACC Cardiovasc Imaging. 2011; 4: 1294-1301 29) Narumi H, Yoshida K, Funabashi N, Hashimoto N, Umehara I, Yoshida S. Abstract 5825: Association between Patterns of FDG Uptake and Arterial Wall Calcification on PET/CT and Atherogenic Risk Factors in Healthy Subjects. Circulation, 2008: S1012 